\title{
The Impact of a Bayesian Penalized-Likelihood Reconstruction Algorithm on a Dual-Time-Point Acquisition Protocol while Performing Ga-68-PSMA PET for Primary Prostate Cancer Detection
}

\author{
Tiago Sampaio Vieira ${ }^{1 *}$, Manuel Teixeira Gomes ${ }^{2}$, Diogo Borges Faria ${ }^{3}$, Sérgio Barroso ${ }^{4}$ and José Pereira de Oliveira ${ }^{1}$ \\ ${ }^{1}$ HPP-Medicina Molecular, Porto, Portugal; Lenitudes Medical Center and Research, Santa Maria da feira, Portugal \\ ${ }^{2}$ Centro Hospitalar do Porto/Hospital de Santo António, Hospital Lusiadas Porto, Porto, Portugal. \\ ${ }^{3}$ HPP-Medicina Molecular, Porto, Portugal; Lenitudes Medical Center and Research, Santa Maria da feira, Portugal \\ School of Health Sciences, University of Aveiro, Portugal \\ ${ }^{4}$ Lenitudes Medical Center and Research, Santa Maria da feira, Portugal
}

\begin{abstract}
A patient performed dual-time-point Ga-68-PSMA PET/CT for prostate cancer evaluation; images reconstructed using OSEM and Q.Clear ${ }^{T M}$. Early and delayed PET revealed focus of increased uptake in prostate right peripheral zone, coincident with a suspicious lesion on MRI; lesion by normal prostate tissue uptake ratios increased with time. Furthermore, delayed-time-point PET revealed another smaller focus of increased uptake in prostate left peripheral zone, not shown by MRI. Some Q.Clear ${ }^{\top \mathrm{TM}} \beta$ values determined higher uptake ratios. Biopsy confirmed two cancer lesions. Optimization of Q.Clear' ${ }^{\mathrm{TM}}$ while performing dual-time-point Ga-68-PSMA PET may further improve imageguided targeted biopsy and focal therapy for primary prostate cancer.
\end{abstract}

Keywords: Dual-time-point Ga-68-PSMA PET; Bayesian penalized likelihood reconstruction algorithm; Primary detection

The Impact of a Bayesian Penalized-Likelihood Reconstruction Algorithm on a Dual-Time-Point Acquisition Protocol while Performing Ga-68-PSMA PET for Primary Prostate Cancer Detection is shown in Figures 1-3

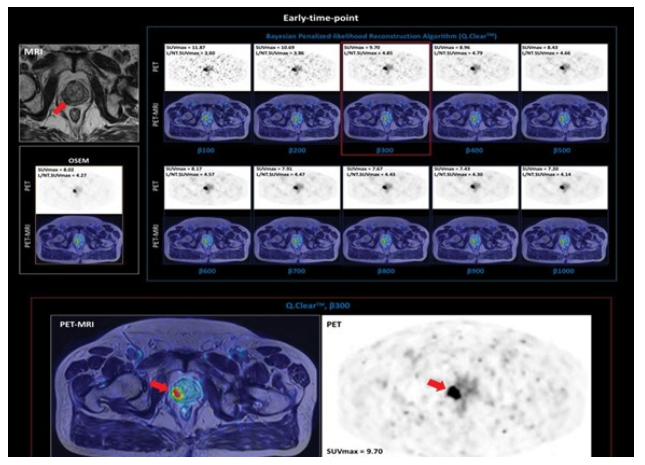

Figure 1: Ga-68-PSMA PET/CT is successfully used for recurrent prostate cancer localization and primary staging in high-risk disease, and targeted biopsy in patients with high suspicion of prostate cancer is also an emerging clinical application [1]. PET may be combined with 3D ultrasound to direct targeted biopsy of the prostate [2]. Innovations on PET scanners and the use of a Bayesian penalized-likelihood reconstruction algorithm (BPLA) such as Q.ClearT ${ }^{\mathrm{TM}}$ can deliver high-quality images, which could further improve the diagnostic accuracy of PET for prostate cancer detection. Q.Clear ${ }^{\mathrm{TM}}$ includes a penalty function, a noise suppression term, controlled by a penalization factor, $B$, allowing an effective convergence, and more accurate SUV quantification in contrast to OSEM, which have to be stopped before contrast convergence to prevent excessive image noise [3-7]. We present the case of a 75 years-old male who performed Ga-68-PSMA PET/CT for prostate cancer evaluation. PET/CT was acquired on a Discovery IQ4R scanner using a dual-time-point acquisition protocol, 1 hour and 2 hours after radiopharmaceutical administration; images were reconstructed applying two methods: OSEM and Q.Clear ${ }^{\top M}$ with $B$ ranging from 100 to 1000 . PET was digitally fused with multiparametric MRI.

Early-time-point (ETP) PET revealed a focus of increased Ga-68-PSMA uptake in prostate right peripheral zone, coincident with the finding of a lesion having 20 $\mathrm{mm}$ of diameter and suspicious for cancer on MRI. The SUV $\mathrm{max}_{\max }$ of this lesion in ETP was higher using Q.Clear ${ }^{T M}$ with $\beta$ of 100 and the ratio of the SUV $V_{\max }$ of the lesion by the SUV $V_{\max }$ of normal prostate tissue L/NT.SUV $\max$ was higher using Q.Clear ${ }^{\mathrm{TM}}$ with $\mathrm{B}$ of 300 .

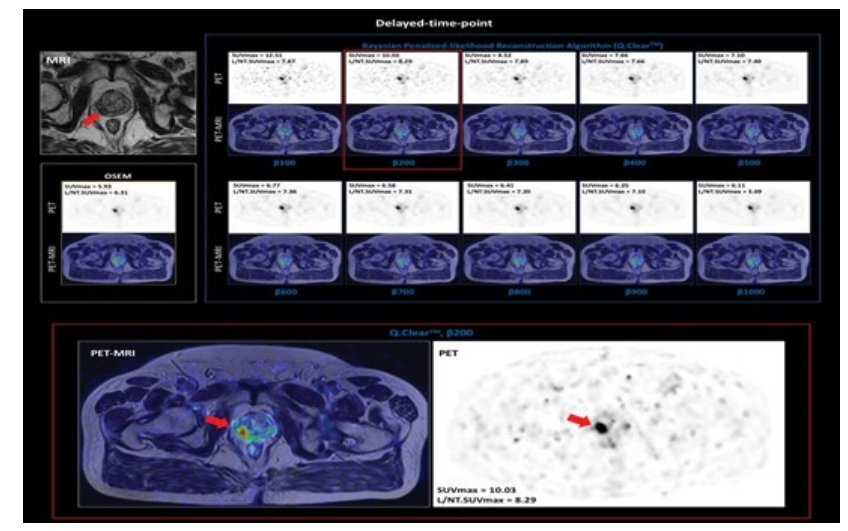

Figure 2: The images of delayed-time-point (DTP) revealed a marked temporal decrease of SUV $\mathrm{Sax}_{\text {in }}$ in normal prostate tissue, resulting in a pronouncedly greater L/NT.SUV $V_{\max }$ in the right peripheral zone lesion on DTP than on ETP. Moreover, on DTP the L/NT.SUV $V_{\max }$ of this lesion was highest when using Q.Clear ${ }^{\mathrm{TM}}$ with $B$ of 200 . This is an interesting finding since previous research revealed that lesion detectability of lung nodules is better when lower $\beta$ values are used, which is thought to relate with lower noise levels in normal pulmonary parenchyma than in tissues of other body regions [6], and as previously referred the uptake in the normal prostatic parenchyma of this patient decreased with time.

${ }^{*}$ Corresponding author: Tiago Sampaio Vieira, HPP-Medicina Molecular Lenitudes Medical Center and Research, Portugal; E-mail: tiago.sampaio.vieira@ gmail.com

Received August 21, 2018; Accepted September 11, 2018; Published September 20, 2018

Citation: Vieira TS, Gomes MT, Faria DB, Barroso S, de Oliveira JP (2018) The Impact of a Bayesian Penalized-Likelihood Reconstruction Algorithm on a DualTime-Point Acquisition Protocol while Performing Ga-68-PSMA PET for Primary Prostate Cancer Detection J Nucl Med Radiat Ther 9: 376. doi: 10.4172/21559619.1000376

Copyright: ( 2018 Vieira TS, et al. This is an open-access article distributed under the terms of the Creative Commons Attribution License, which permits unrestricted use, distribution, and reproduction in any medium, provided the original author and source are credited. 
Citation: Vieira TS, Gomes MT, Faria DB, Barroso S, de Oliveira JP (2018) The Impact of a Bayesian Penalized-Likelihood Reconstruction Algorithm on a Dual-Time-Point Acquisition Protocol while Performing Ga-68-PSMA PET for Primary Prostate Cancer Detection J Nucl Med Radiat Ther 9: 376. doi: 10.4172/2155-9619.1000376

Page 2 of 2

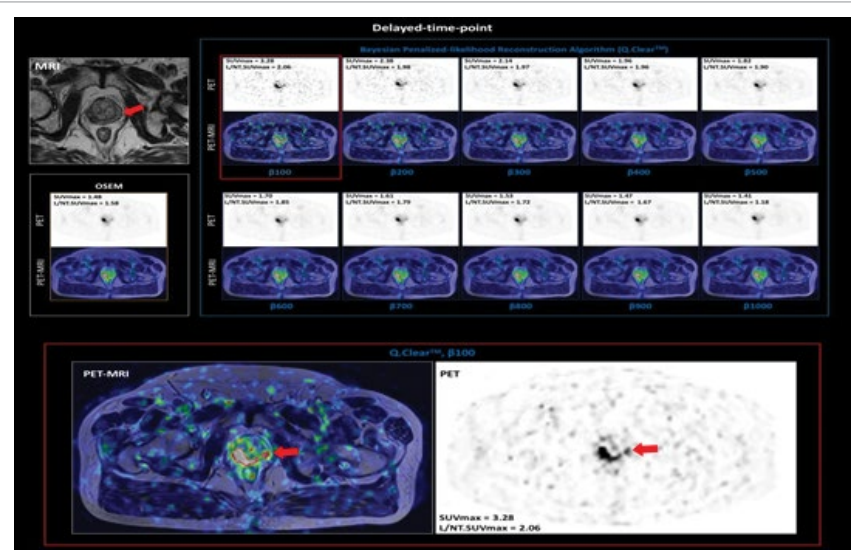

Figure 3: DTP PET revealed another smaller focus of increased uptake in prostate left peripheral zone, and L/NT.SUV $\mathrm{max}_{\max }$ of this focus was higher when using $B$ of 100 . This second focus wasn't coincident with any observable abnormality on MRI, but a biopsy confirmed the existence of two prostate cancer lesions, one in each lateral peripheral zone. In fact, Ga-68-PSMA PET/MRI proved to improve the diagnostic accuracy for primary prostate cancer [8,9], the value of DTP Ga-68PSMA PET for the detection of primary and recurrent prostate cancer lesions has been described $[10,11]$ and the synergistic effect of BPLA and DTP on recurrent prostate cancer detection was also shown [12]. This case suggests that the optimization of a BPLA while performing dual-time-point Ga-68-PSMA PET may further improve the capabilities of multimodality image-guided targeted-biopsy for primary prostate cancer detection and for focal therapy planning.

\section{References}

1. Fendler WP, Eiber M, Beheshti M, Bomanji J, Ceci F, et al. (2017) 68Ga-PSMA PET/CT: Joint EANM and SNMMI procedure guideline for prostate cancer imaging: Version 1.0, Eur J Nucl Med Mol Imaging 44: 1014-1024.

2. Fei B, Nieh PT, Schuster DM, Master VA (2013) PET-directed, 3D Ultrasound guided prostate biopsy Diagn Imaging Eur 29: 12-15.

3. Mumcuoglu EU, Leahy R, Cherry SR, Zhou Z (1994) Fast gradient-based methods for bayesian reconstruction of transmission and emission PET images, IEEE Transactions Med Imaging 13: 687-701.

4. Asma E, Ahn S, Ross SG, Manjeshwar R (2012) Accurate and consistent lesion quantitation with clinically acceptable penalized likelihood images. 2012 IEEE nuclear science symposium and medical imaging conference record (NSS/ MIC) 4062-4066.

5. Parvizi N, Franklin JM, McGowan DR, Teoh EJ, Bradley KM, et al. (2015) Does a novel penalized likelihood reconstruction of 18F-FDG PET-CT improve signalto-background in colorectal liver metastases? Eur J Radiol 84: 1873-1878.

6. Teoh EJ, McGowan DR, Macpherson RE, Bradley KM, Gleeson FV, et al. (2015) Phantom and clinical evaluation of the bayesian penalized likelihood reconstruction algorithm Q.Clear on an LYSO PET/CT System. J Nucl Med 56 1447-1452.

7. Reynes-Llompart G, Gamez-Cenzano C, Romero-Zayas I, Rodríguez-Be $\mathrm{L}$, Vercher-Conejero JL, et al. (2017) Performance characteristics of the wholebody discovery iq pet/ct system. J Nucl Med 58: 1155-1161.

8. Eiber M, Weirich G, Holzapfel K (2016) Simultaneous 68Ga-PSMA HBED-CC PET/MRI Improves the localization of primary prostate cancer. Eur Urol 70 829-836.

9. Zamboglou C, Drendel V, Jilg CA, Rischke HC, Beck TI, et al. (2017) Comparison of (68)Ga-HBED-CC PSMA-PET/CT and multiparametric MRI fo gross tumour volume detection in patients with primary prostate cancer based on slice by slice comparison with histopathology. Theranostics 7: 228-237.

10. Afshar-Oromieh A, Malcher A, Eder M, Eisenhut M, Linhart HG, et al. (2013) $\mathrm{PET}$ imaging with a $[68 \mathrm{Ga}$ ] gallium-labelled PSMA ligand for the diagnosis of prostate cancer: Biodistribution in humans and first evaluation of tumour lesions. Eur J Nucl Med Mol Imaging 40: 486-495.

11. Sahlmann CO, Meller B, Bouter C, Ritter CO, Ströbel P, et al. (2016) Biphasic ${ }^{68} \mathrm{Ga}-\mathrm{PSMA}$-HBED-CC-PET/CT in patients with recurrent and high-risk prostate carcinoma. Eur J Nucl Med Mol Imaging 43: 898-905

12. Vieira ST, Faria DB, Silva AF (2018) The impact of a bayesian penalizedlikelihood reconstruction algorithm on delayed-time-point Ga-68-PSMA PET for improved recurrent prostate cancer detection. Eur J Nucl Med Mol Imaging 45: 1461-1462. 